\title{
Estructuras semánticas de problemas aditivos de enunciado verbal en libros de texto mexicanos
}

\section{Semantic structures of additive problems of verbal statement in Mexican textbooks}

\section{Camilo Andrés Rodríguez-Nieto ${ }^{1}$ \\ Catalina Navarro Sandoval ${ }^{2}$ \\ Angela Nolfa Castro Inostroza ${ }^{3}$ \\ María del Socorro García González ${ }^{4}$}

\begin{abstract}
Resumen: El objetivo de esta investigación es caracterizar los problemas aditivos de enunciado verbal presentes en los libros de texto del segundo periodo escolar de Educación básica en México (primer, segundo y tercer grados) con base en su estructura semántica. Bajo un enfoque cualitativo, se realizó un análisis de contenido documental, en el que se establecieron unidades y categorías de análisis. Los resultados sugieren que los libros de texto analizados presentan en su mayoría, problemas con estructuras sencillas de resolver como las de cambio y combinación, y pocos problemas con estructuras complejas como las de comparación e igualación. Identificando además algunas estructuras semánticas compuestas, éstas últimas representan mayor desafío para la resolución de problemas.
\end{abstract}

Fecha de recepción: 18 de enero de 2019. Fecha de aceptación: 23 de mayo de 2019.

${ }^{1}$ Estudiante del Doctorado en Ciencias con Especialidad en Matemática Educativa CIMATE, Universidad Autónoma de Guerrero (UAGro), México. camilorodriguez274@gmail.com, orcid.org/0000-0001-9922-4079

2 Doctora en Ciencias con Especialidad en Matemática Educativa por la Universidad Autónoma de Guerrero, México. Profesora titular CIMATE, Universidad Autónoma de Guerrero (UAGro), México.nasacamx@yahoo.com.mx, orcid.org/0000-0001-5214-0062

3 Doctora en Educación en el ámbito de Didáctica de la Matemática por la Universidad Autónoma de Barcelona (UAB). Docente Centro de Docencia Superior en Ciencias Básicas, Universidad Austral de Chile (UACh), Sede Puerto Montt, Chile. angela.castro@uach.cl, orcid.org/0000-0002-1732-6520

4 Doctora en Matemática Educativa por el Centro de Investigación y de Estudios Avanzados del Instituto Politécnico Nacional (Cinvestav-IPN). Profesora titular CIMATE, Universidad Autónoma de Guerrero (UAGro), México. mgargonza@gmail.com,orcid.org/0000-0001-7088-1075 
Palabras clave: problemas aditivos, estructura semántica, libro de texto.

\begin{abstract}
The research aim is to characterize the additive problems of verbal statement in the textbooks of the second period of basic education in Mexico (first, second and third grades) according to their semantic structure. From the qualitative approach, an analysis of documentary content was carried out, in which units and categories of analysis were established. The results suggest that the analyzed textbooks mostly present problems with simple structures to solve such as change and combination, and just a few of them with complex structures such as comparison and equalization. Also identifying some composite semantic structures, that represent a challenge in problem solving.
\end{abstract}

Keywords: additive problems, semantic structure, textbook.

\title{
1. INTRODUCCIÓN
}

Con el propósito de formar estudiantes matemáticamente competentes, en la enseñanza y aprendizaje de las matemáticas, la resolución de problemas se ha considerado como una actividad de primer orden y eje central para el desarrollo de contenidos matemáticos (NTCM, 2000; García, 2011; CCSSI, 2018). La resolución de problemas es una competencia que los estudiantes deben desarrollar de manera autónoma, con la intención que éstos identifiquen, planteen y resuelvan una variedad de problemas o situaciones cotidianas en las que se consideren diversos tipos de soluciones, o en las que deban identificar cuando sobran o faltan datos en el enunciado del problema (SEP, 2011a). Sin embargo, pruebas estandarizadas como PISA, evidencian que más de la mitad de los estudiantes que rinden esta evaluación, en matemáticas no alcanzan el nivel básico de competencias (nivel 2) ${ }^{5}$ (OCDE, 2016). A nivel nacional, la prueba PLANEA ${ }^{6}$ reportó que en el puntaje nacional de aciertos los estudiantes alcanzaron un

5 Realizar procedimientos rutinarios, ejercicios, tales como operaciones aritméticas en situaciones en donde todas las instrucciones se les son dadas, pero tienen problemas identificando cómo una situación del mundo real puede ser representada matemáticamente (OCDE, 2016).

6 PLANEA (Plan Nacional de Evaluación de los Aprendizajes). La cual "evalúa los aprendizajes clave que los alumnos de cuarto grado de Educación primaria han alcanzado durante su trayecto escolar en Español y Matemáticas" (INEE, 2016, p.5). 
nivel bajo en la resolución de problemas aditivos, cuando éstos no tienen la incógnita en la cantidad final (INEE,7 2016).

En particular, la resolución de problemas aditivos ha sido estudiada a partir de su estructura semántica y la componente sintáctica, distinguiendo tipos de problemas cuando se enfatiza en las relaciones entre los elementos de un enunciado y los significados de las palabras (Van Dijk y Kintsch, 1983; Riley y Greeno, 1988). Asimismo, la resolución de problemas se ha desarrollado a partir de estrategias y procesos heurísticos, como, por ejemplo, comprender el problema, concebir un plan, ejecutar el plan y la visión retrospectiva (Pólya, 1989), lo que ha permitido el surgimiento de las estrategias cognitivas para resolver problemas (Schoenfeld, 1985). Considerando el grado de dificultad (cantidad de información y relaciones entre los datos), existen otras formas de resolver problemas aritméticos, Verschaffel, Greer y De Corte (2000) propusieron dos modos de resolución: el genuino, utilizado para resolver problemas considerados difíciles desde un contexto situacional, y el superficial usado para resolver problemas más fáciles.

Desde la literatura, algunas investigaciones ponen de manifiesto que los estudiantes al resolver problemas aditivos utilizan estrategias superficiales como, por ejemplo, el paso directo de la información contenida en el problema a una operación para obtener la solución sin comprender el enunciado (Verschaffel y De Corte, 1993; Blanco, Caballero y Cárdenas, 2014; Chamoso, Vicente, Manchado y Múñez, 2014; Ortíz, 2014), malinterpretan el enunciado del problema (Orrantia, González y Vicente, 2005; Polotskaia, Savard y Freiman, 2016), escasa comprensión del problema (García-García, Navarro y Rodríguez-Vásquez, 2014), y eligen una operación diferente a la adecuada para la resolución del problema (Butto y Martínez, 2012).

El uso de estrategias superficiales, en muchos casos es promovido por los maestros y los materiales curriculares. En muchas ocasiones, los profesores de matemáticas utilizan palabras clave en el discurso para enseñar a sus estudiantes a resolver un problema (García-García, 2014), proponen problemas aditivos verbales obviando las clasificaciones según la estructura semántica, utilizando estructuras sencillas de resolver (Castro, Gorgorió y Prat, 2014), y regularmente al revisar resultados no se atienden los procedimientos realizados por los estudiantes (Polotskaia et al., 2016). Se podría pensar, que dado que los materiales curriculares como el libro de texto, han desempeñado un papel fundamental en el proceso de enseñanza y aprendizaje de las matemáticas, estas prácticas son

\footnotetext{
7 Instituto Nacional para la Evaluación de la Educación.
} 
influenciadas, de algún modo, por los contenidos y el tipo de problemas presentes en libros de texto escolares de matemáticas, en los que en muchas ocasiones, se proponen problemas poco desafiantes y cuyo enunciado presenta poca variedad en el lugar de la incógnita (Orrantia et al., 2005; Chamoso et al., 2014; Ortíz, 2014; Sánchez y Vicente, 2015).

El libro de texto se ha considerado una herramienta importante para la planificación de clases por parte de los profesores (Azcárate y Serradó, 2006; Pepin, Gueudet y Trouche, 2013; Aké y Godino, 2018). No obstante, es importante el uso que se le dé al libro de texto, pues de éste dependerá la influencia favorable o desfavorable durante la enseñanza de cualquier contenido. En algunos casos los libros de texto presentan errores en los contenidos, lo que podría causar inconsistencias durante el proceso de razonamiento y aprendizaje de estudiantes y en la enseñanza por parte de los profesores (Jaime, Chapa y Gutiérrez, 1992). De ahí la importancia de realizar investigaciones centradas en el análisis de libros de texto escolares, para evaluar su pertinencia y adecuación de sus contenidos (Font y Godino, 2006).

En esta línea, diferentes investigaciones han estudiado los problemas aditivos de enunciado verbal desde su estructura semántica y sintáctica. Dichos estudios han reportado diferentes tipos de problemas aditivos según su estructura (Heller y Greeno, 1978; Bermejo y Rodríguez, 1987; Orrantia et al., 2005; Chamoso et al., 2014; Ortiz, 2014), han enfatizado sobre el grado de dificultad que involucra este tipo de problemas (Carpenter, Hiebert y Moser, 1981) y los niveles de conocimiento aritmético asociados a un determinado tipo de problema aditivo (Nesher, Greeno y Riley, 1982; Nesher, 1999).

En el contexto de la reforma 2011 en México, el plan y programas de estudios de la Educación básica señalan que los estudiantes al culminar el segundo periodo escolar (1ㅇ-은) saben resolver problemas aditivos con diferente estructura, desarrollar formas de pensar, utilizar diferentes técnicas o recursos y procedimientos eficientes para la resolución de problemas (SEP, 2011a). A nivel nacional, en México se realizó una clasificación de problemas aditivos de libros de correspondientes a las reformas de los 60, 70 y 90, encontrándose problemas de dos etapas y en su mayoría se proponían problemas con estructuras de cambio y combinación (Aguillón y Resendiz, 2012).

En este sentido, tras el paso de varias reformas educativas, se pretende saber qué tipo de problemas emplearían los profesores en las clases de matemáticas para desarrollar una competencia de resolución de problemas en los estudiantes, para ello es necesario analizar los libros de texto de la reforma 
actual para identificar la promoción de los problemas aditivos que involucran las diferentes estructuras semánticas. Por lo tanto, en este estudio se propuso caracterizar las estructuras semánticas y componente sintáctica de los problemas aditivos de enunciado verbal en los libros de texto desafíos matemáticos del segundo periodo escolar de la educación básica en México.

\section{MARCO CONCEPTUAL}

\subsection{Problemas aditivos de enunciado verbal}

Un problema es toda situación desafiante o retadora en la que hay un planteamiento inicial y una exigencia que obliga a transformarla. La vía de solución es desconocida o no tiene un camino de solución inmediato y la persona quiere realmente realizar la transformación (Rizo y Campistrous, 1999). Si éstas dos últimas condiciones no se dan, la situación no constituye un problema para la persona.

En la mayoría de los currículos educativos, la resolución de problemas se presenta como una competencia basada en que el individuo resuelva de manera autónoma, tareas matemáticas desafiantes (SEP, 2011b; CCSSI, 2018). La resolución de problemas no sólo se centra en situaciones de la vida cotidiana, sino que se preocupa por aquellas situaciones que no resulten familiares, que tengan un alto grado de dificultad, donde el individuo manifieste sus habilidades cognitivas (Echenique, 2006).

Existen distintos tipos de problemas matemáticos, entre los que se encuentran los aritméticos, que:

En su enunciado presentan datos en forma de cantidades y establecen entre ellos relaciones de tipo cuantitativo, cuyas preguntas hacen referencia a la determinación de una o varias cantidades o a sus relaciones, y que necesitan la realización de operaciones aritméticas para su resolución (Echenique, 2006, p.30).

Los problemas aritméticos pueden ser: aditivos, multiplicativos, entre otros. Asimismo, estos problemas se pueden presentar de diversas formas, por ejemplo, verbal, numérica y gráfica (Bonilla, Sánchez y Guerrero, 1999).

En este estudio son de interés los problemas aditivos (aditivo-sustractivos), los cuales se caracterizan por requerir en su resolución una o varias adiciones y/o sustracciones (Vergnaud, 1991; Echenique, 2006). También, este tipo de 
problemas poseen relaciones formadas por adiciones o sustracciones, a lo que se le conoce como estructuras aditivas (Castro, Rico y Castro, 1995; Bonilla et al., 1999). Cañadas y Castro (2011) mencionan que los problemas de una etapa o simples son aquellos que se resuelven mediante una única operación aritmética, ya sea una adición o una sustracción. Los problemas simples se constituyen de una estructura simple. Mientras que los problemas de más de una etapa o de $n$ etapas, son los que necesitan $n$ operaciones para llegar a su solución. Así, los problemas aditivos de una etapa requieren de una sola suma o resta para su resolución y los de $n$ etapas implican $n$ sumas y/o restas.

Los problemas aditivos verbales se estudiaron desde las relaciones aditivas ternarias, utilizándose seis categorías con sus esquemas ${ }^{8}$ basados en relacionar y operar con medidas (Durand y Vergnaud, 1976; Vergnaud, 1991). También, los problemas aditivos de enunciado verbal se clasifican con base en la estructura semántica, es decir, las relaciones entre los elementos que aparecen en el enunciado de la situación problema (Van Dijk y Kintsch, 1983; Orrantia, 2003; Orrantia et al., 2005; Castro et al., 2014). Cabe mencionar que, la semántica desde una perspectiva léxica, estudia el significado de las palabras, así como las diversas relaciones de sentido que se establecen entre ellas (Real Academia Española, 2014).

\subsection{Clasificación de PROBlemas aritmÉticos de ENUNCIAdO VERBAL (PAEV) ADITIVOS (PAEV ADITIVOS)}

La clasificación de los PAEV aditivos se realizó con base en los trabajos de Heller y Greeno (1978), Orrantia et al. (2005) y Cañadas y Castro (2011), a partir de su estructura semántica y la componente sintáctica en cuatro tipos de problemas: cambio, combinación, comparación e igualación.

En la estructura de cambio se distinguen tres elementos diferentes, una cantidad inicial sometida a una transformación (cambio) que la modifica para llegar a una cantidad final. El efecto del cambio puede ser un aumento o una disminución. En la estructura de combinación se consideran dos cantidades que forman parte de un todo que las incluye en su totalidad. Lo desconocido puede ser el conjunto o cantidad total o uno de los subconjuntos. La estructura

\footnotetext{
8 Un esquema en el sentido de Orrantia et al. (2005) es una representación gráfica del problema que permite operar con las relaciones semánticas implícitas en el enunciado.
} 
de comparación parte de dos cantidades independientes que se relacionan mediante la comparación, en este tipo de problemas se relacionan tres cantidades, el referente (R), el comparado (C) y la diferencia (D). La relación de comparación está dada por palabras que están presentes en el enunciado del problema, como, por ejemplo, más que y menos que (Riley, Greeno y Heller, 1983; Castro, Rico y Castro, 1995; Orrantia et al., 2005; Cañadas y Castro, 2011).

A esta clasificación se le agrega una cuarta categoría, correspondiente a la estructura semántica de igualación, identificada como aquella que restringe lo desconocido a la diferencia entre: cantidad dada y la cantidad deseada. Esta estructura se compone de tres cantidades, igualación, comparado y referente. Dichos problemas en el enunciado exponen una acción física necesaria para que una cantidad sea igual a otra (Cañadas y Castro, 2011). Otros investigadores como Castro et al. (1995), Echenique (2006) y Orrantia (2006) mencionan que, los problemas de igualación se originaron a partir de una relación entre los problemas de cambio y comparación, donde se produce una acción al comparar dos cantidades y luego un cambio de aumento o disminución respecto de una cantidad. Cabe resaltar que, los problemas de cambio e igualación se consideran dinámicos y los problemas de combinación y comparación son estáticos.

La clasificación de los problemas aditivos se extiende a veinte tipos (ver Tabla 1), cuando se hace referencia a la componente sintáctica, entendida como el orden y las relaciones entre los datos, palabras, símbolos y el lugar que ocupa la incógnita (cantidad desconocida) en el enunciado del problema (Puig y Cerdán, 1988).

Además, según el grado de dificultad los PAEV aditivos, se pueden clasificar en consistentes e inconsistentes. Los consistentes hacen referencia a los problemas que se solucionan por medio de una estrategia de traducción directa o modelado directo, es decir, los términos del enunciado o palabra clave, por ejemplo, "ganar" o "más que", coinciden con la operación a realizar, en este caso la adición (Hegarty, Mayer y Monk, 1995; Orrantia et al., 2005). Los inconsistentes presentan un mayor grado de dificultad, no pueden solucionarse por medio de modelado directo, es decir, los términos o palabras clave entran en conflicto con la operación a realizar, por ejemplo, "ganar" o "más que" y se requiere de una sustracción (Hegarty et al., 1995; Orrantia et al., 2005). 
Tabla 1. Componente sintáctica basada en el orden y lugar de la incógnita en el enunciado del PAEV aditivo.

\begin{tabular}{|c|c|c|}
\hline Estructura semántica & Descr & e la componente sintáctica \\
\hline \multirow{6}{*}{ Cambio } & \multirow{3}{*}{ Aumento } & Incógnita en la cantidad final. \\
\hline & & Incógnita en la cantidad de cambio. \\
\hline & & Incógnita en la cantidad inicial. \\
\hline & \multirow{3}{*}{ Disminución } & Incógnita en la cantidad final. \\
\hline & & Incógnita en la cantidad de cambio. \\
\hline & & Incógnita en la cantidad inicial. \\
\hline \multirow{2}{*}{ Combinación } & \multicolumn{2}{|c|}{ Incógnita en la cantidad total } \\
\hline & \multicolumn{2}{|c|}{ Incógnita en una de las partes que conforman el todo. } \\
\hline \multirow{6}{*}{ Comparación } & \multirow{3}{*}{ Aumento } & Incógnita en la diferencia. \\
\hline & & Incógnita en el comparado. \\
\hline & & Incógnita en el referente. \\
\hline & \multirow{3}{*}{ Disminución } & Incógnita en la diferencia. \\
\hline & & Incógnita en el comparado. \\
\hline & & Incógnita en el referente. \\
\hline \multirow{6}{*}{ Igualación } & \multirow{3}{*}{ Aumento } & Incógnita en la igualación. \\
\hline & & Incógnita en el comparado. \\
\hline & & Incógnita en el referente. \\
\hline & \multirow{3}{*}{ Disminución } & Incógnita en la igualación. \\
\hline & & Incógnita en el comparado. \\
\hline & & Incógnita en el referente. \\
\hline
\end{tabular}

Nota. Información adaptada de Carpenter et al. (1981), Puig y Cerdán (1988), Orrantia et al. (2005) y Cañadas y Castro (2011).

Los problemas que presentan mayor dificultad se constituyen de estructuras complejas asociadas a los problemas inconsistentes identificándose esquemas parte-parte-todo donde se relacionan tres componentes que aparecen en el enunciado del problema mediante sumas o restas usando la reversibilidad para comparar y establecer igualdades y desigualdades (Nesher, 1999; Castro, 2013; 
Orrantia et al., 2005). En este sentido, para resolver un problema con estructuras complejas se requiere el uso de estrategias para comprender el enunciado y trasladar adecuadamente la información escrita a una representación abstracta o a un esquema donde se tengan en cuenta las relaciones semánticas accediendo al conocimiento conceptual (Riley et al., 1983; Orrantia et al., 2005).

Las estructuras semánticas compuestas dependen de las estructuras simples y el número de etapas requeridas para la resolución de un problema. En este sentido, por cada operación que se utilice, va de manera implícita una estructura semántica simple (cambio, combinación, comparación e igualación) y sus respectivas tipologías según la componente sintáctica.

\section{METODOLOGÍA}

La investigación adoptó una metodología de tipo cualitativa descriptiva, mediante el análisis de contenido de acuerdo con López-Noguero (2002) quien señala que éste es un proceso descriptivo para organizar y analizar documentos, entre los que se destacan los textos científicos, artículos, libros, planes de estudios, leyes, fotografías, entre otros. Para llevar a cabo un análisis documental es importante que desde el principio el investigador revise y conozca la documentación referente al problema que se está desarrollando. Puesto que el interés del trabajo es caracterizar y describir la tipología de problemas aditivos de enunciado verbal en libros de texto.

Este trabajo se llevó a cabo en tres fases, en la primera se realizó una revisión de planes y programas de estudio (SEP, 2011a, 2011b) y libros de texto (SEP, 2016a, 2016b, 2016c), propuestos por la Secretaría de Educación Pública, con el propósito de identificar donde se inicia la resolución de problemas aditivos en el nivel básico. Como resultado de la fase anterior, en una segunda fase se seleccionaron tres libros de texto correspondientes al segundo periodo escolar y en la tercera fase se realizó la caracterización de los PAEV aditivos, por medio de un análisis de contenido documental.

\subsection{REVISIÓN DE LOS PLANES Y PROGRAMAS DE ESTUDIOS}

La revisión de los planes y programas de estudio, permitió identificar la promoción de los problemas aditivos de enunciado verbal en la educación básica. En 
este sentido, se tuvieron en cuenta los ejes temáticos, el tema de problemas aditivos y la afirmación de los programas de estudio que al culminar el segundo periodo escolar los estudiantes saben resolver problemas aditivos con diferentes estructuras (SEP, 2011a). Por tanto, el estudio se centró, además, en revisar los libros de texto de educación primaria para conocer si la tipología de problemas mantenía una diversidad de estructuras.

A partir de ello, se identificaron tipos de problemas aditivos que se presentan en los libros de texto, diferenciando entre problemas aditivos verbales, numéricos, gráficos desde la perspectiva de Bonilla et al. (1999), así como aquellos que se constituyen de más de dos tipos, por ejemplo, numérico-gráfico. Este primer análisis nos permitió observar que, los problemas aditivos de tipo numérico son los que se presentan con mayor frecuencia en los libros de texto analizados. Respecto de los problemas aditivos verbales se identificaron en el primer grado veintitrés, en segundo grado veintinueve y en tercer grado dieciocho, en este último libro se presentan además problemas de enunciado verbal con estructuras multiplicativas. En este estudio, se consideraron solamente problemas verbales y de éstos se obtuvieron los PAEV aditivos, pues la literatura dejó entrever que las dificultades de estudiantes y profesores se centran en el escaso dominio conceptual de los problemas antes mencionados.

\subsection{SELECCIÓN Y DESCRIPCIÓN DE LOS LIBROS DE TEXTO}

La selección de los libros de texto, se dio a partir de la revisión de los planes y programas de estudio y los textos escolares de educación primaria en México. Al identificar en el plan de estudios que al culminar el segundo periodo escolar los estudiantes deberían resolver problemas aditivos con diferentes estructuras, nos tomamos la tarea de seleccionar y analizar los textos correspondientes al primero, segundo y tercer grado. Estos libros son materiales gratuitos y únicos elaborados por la Comisión Nacional de Libros de Textos Gratuitos (CONALITEG) correspondientes a la tercera reimpresión del año 2016 (ciclo escolar 2017-2018), los cuales son ofrecidos por la SEP en sus versiones para el estudiante y para el profesor usados por escuelas públicas y privadas de México.

Los libros de texto "desafíos matemáticos" poseen consignas que contienen actividades donde el estudiante debe buscar estrategias que le ayudan a ganar, crear espacios de interacción con sus compañeros para vencer los desafíos, y conseguir estrategias para solucionar problemas. Asimismo, este material curricular 
está apegado al programa oficial y cubre todos sus contenidos, tiene un formato ágil y desafíos que apoyan a la labor diaria del docente, para que éste los analice previamente a su puesta en práctica en el aula (SEP, 2016a; 2016b; 2016c, p.7).

Los libros de texto de primero, segundo y tercer grados están constituidos por bloques, que contienen a los desafíos y, éstos a las consignas donde se presentan actividades que contienen problemas matemáticos, así como las instrucciones o la forma de organizar las actividades (SEP, 2016a; 2016b; 2016c). En los bloques se incluyen contenidos de los tres ejes temático: sentido numérico y pensamiento algebraico, forma, espacio y medida y manejo de la información, con la intención de estudiarlos simultáneamente para con ello tener una visión global de las matemáticas (SEP, 2011a). Cabe señalar que, en cada bloque se presentan los aprendizajes esperados de los diferentes ejes temáticos, los que resultan útiles a los docentes para llevar a cabo un proceso de evaluación y de apoyo para el logro de aprendizajes (SEP, 2011b). En la Tabla 2 se describen los libros con respecto a los problemas aditivos.

Tabla 2. Descripción de los libros de texto y desafíos que proponen problemas aditivos.

\begin{tabular}{ccccc}
\hline $\begin{array}{c}\text { Libro de } \\
\text { texto }\end{array}$ & Desafíos & $\begin{array}{c}\text { Desafíos que proponen } \\
\text { problemas aditivos }\end{array}$ & $\begin{array}{c}\text { Cantidad de problemas } \\
\text { aditivos }\end{array}$ & $\begin{array}{c}\text { Cantidad de PAEV } \\
\text { aditivos }\end{array}$ \\
\hline $1^{\circ}$ & 57 & 32 & $116(100 \%)$ & $23(19.8 \%)$ \\
$2^{\circ}$ & 59 & 21 & $104(100 \%)$ & $29(27.9 \%)$ \\
$3^{\circ}$ & 76 & 14 & $104(100 \%)$ & $18(17.3 \%)$ \\
\hline
\end{tabular}

Nota: información tomada de los libros de texto del segundo periodo escolar (SEP, 2016a; 2016b; 2016c).

\subsection{ANÁLISIS DE CONTENIDO}

Esta investigación se realiza con base en el análisis de contenido desde la perspectiva de López-Noguero (2002) como una forma particular de análisis de documentos, con la que no se pretende analizar el estilo del texto, sino las ideas expresadas en él, como por ejemplo, el significado de las palabras, temas o frases que requieren de una cuantificación. Bajo este contexto analizamos los PAEV aditivos presentes en los libros de texto, de acuerdo con su estructura semántica y componente sintáctica. Para ello, se consideran elementos reportados en 
Navarro (2015), para establecer y definir las unidades de análisis, y determinar las categorías que representan las variables de la investigación.

\subsubsection{UNIDADES Y CATEGORÍAS DE ANÁLISIS}

Con base en los elementos teóricos y estudios realizados sobre análisis de libros de texto, se consideraron las unidades y categorías de análisis a partir de la clasificación para los PAEV aditivos de acuerdo con su estructura semántica desde la perspectiva de Riley et al. (1983), Orrantia et al. (2005), y Cañadas y Castro (2011), quienes han clasificado a los PAEV aditivos en: cambio, combinación, comparación e igualación (ver Figura 1). Las categorías de análisis se conformaron a partir de la componente sintáctica, siendo los elementos o dimensiones de las variables investigadas y son útiles para clasificar o agrupar dependiendo de las unidades de análisis (López-Noguero, 2002).

Figura 1. Unidades y categorías de análisis basadas en la estructura semántica y componente sintáctica.

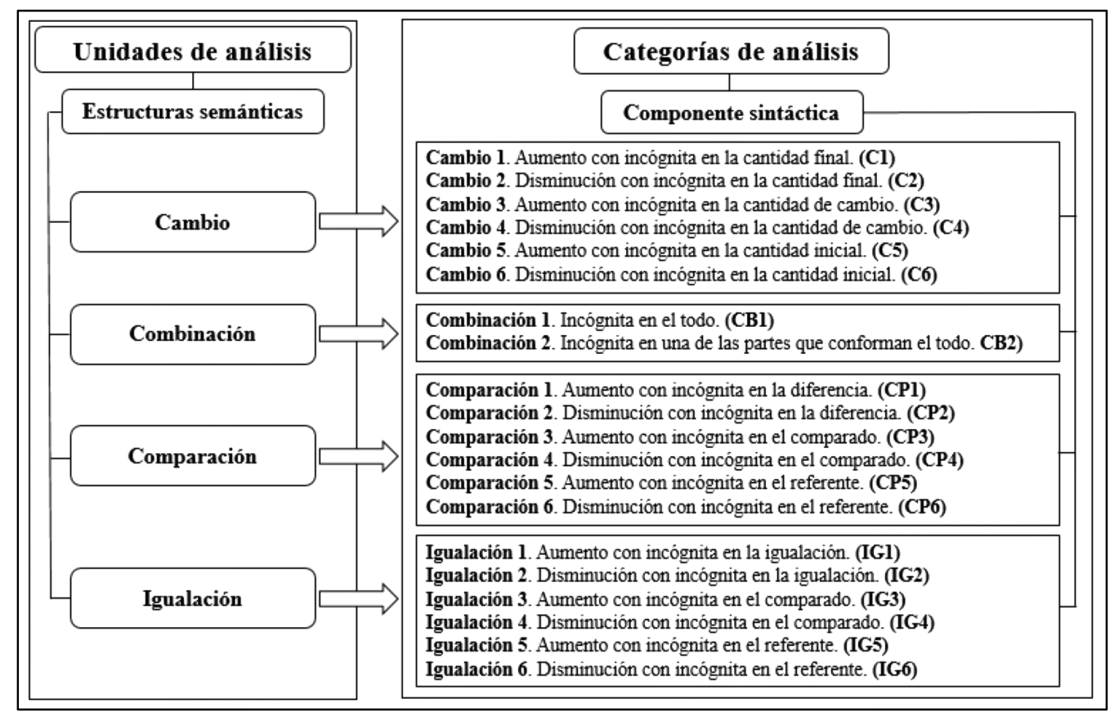

Nota: Información adaptada de Carpenter et al. (1981), Puig y Cerdán (1988), Orrantia et al. (2005) y Cañadas y Castro (2011). 
Por otra parte, Ios PAEV aditivos de más de una etapa, se analizan a partir de las estructuras compuestas. Cuando los problemas requieren para su solución más de una operación, debe asociarse una estructura semántica simple por cada etapa. Por ejemplo, combinación 1-cambio 2 (CB1-C2).

\section{ANÁLISIS Y RESULTADOS}

Con base en las unidades y categorías de análisis establecidas en la metodología, se presentan los resultados de esta investigación y la operatividad de los elementos teóricos considerados, donde se dan a conocer la variedad de estructuras semánticas contenidas en los PAEV aditivos de los libros de texto correspondientes al segundo período escolar. En concreto, se presenta el análisis y resultados en tres secciones de cada libro de texto y, luego de forma general mediante una tabla sintetizando la cantidad de estructuras semánticas simples y compuestas.

\subsection{PAEV ADITIVOS EN EL LIBRO DE PRIMER GRADO}

En la Tabla 3, se evidencian los desafíos que contienen PAEV aditivos, los cuales se tendrán en cuenta en el análisis. Cabe resaltar que, el libro de texto de primer grado para el maestro en algunos casos hace referencia a determinar el resultado al "juntar" o "separar" colecciones de objetos (SEP, 2016a), siendo éste un significado asociado a la adición y a la estructura semántica de combinación. Sin embargo, se presentan otras estructuras como las de cambio. 
Tabla 3. Desafíos que contienen PAEV aditivos en el libro de primer grado.

\begin{tabular}{cccc}
\hline Bloque & Desafío & Consignas & Título \\
\hline I & 13 & 2 & ¿Cómo quedó? \\
\hline \multirow{3}{*}{$\|$} & 22 & 1 & ¿Cuánto cambio queda? \\
\cline { 2 - 4 } & 24 & 1 & El camión \\
& 27 & 2 & ¿Hay alguna mal? \\
III & 28 & 1 & ¿Cuándo usar,,$+-=$ ? \\
& 35 & 1 & Historias con números \\
IV & 36 & 1 & Las granjas \\
\hline
\end{tabular}

Teniendo en cuenta que los desafíos están conformados por consignas y éstas contienen a los problemas, se presenta el desafío 13, conformado por dos consignas que reúnen cinco PAEV aditivos (ver Figura 2), pertenecientes al contenido: obtención del resultado de agregar o quitar elementos de una colección, juntar o separar colecciones, buscar lo que le falta a una cierta cantidad para llegar a otra, y avanzar o retroceder en una sucesión.

Figura 2. Consigna 1 y 2 del desafío 13 ¿Cómo quedó? (SEP, 2016a, p.42).

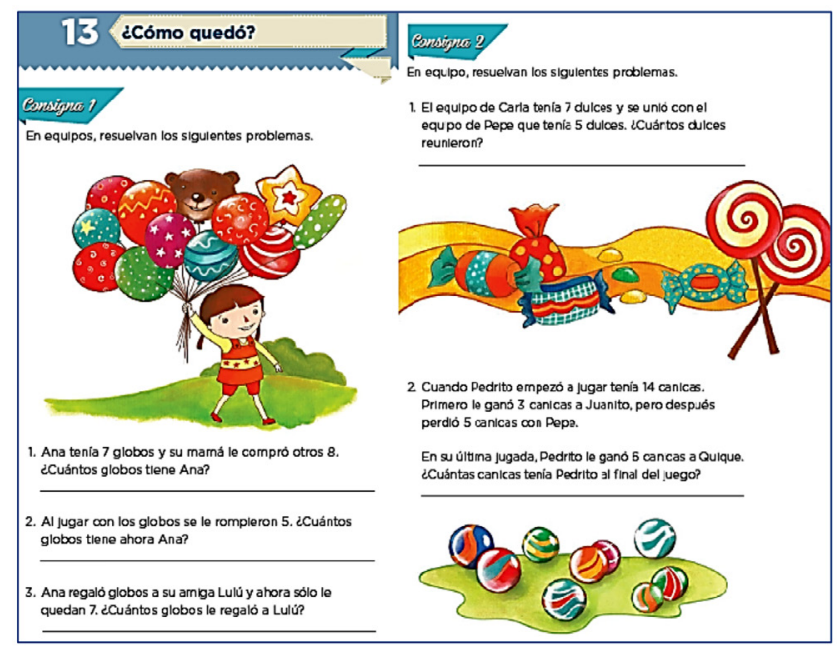


En el primer problema se evidencia una estructura semántica de cambio 1 aumento con la incógnita en la cantidad final. El segundo problema se constituye de una estructura semántica de cambio 2 con la incógnita en la cantidad final. El tercer problema tiene una estructura semántica de cambio 4 con la incógnita en la cantidad de cambio. De forma general, este tipo de problemas son de tipo consistente, de tal menara que, los datos se presentan ordenados y se pueden resolver por un método de resolución directo. Cabe resaltar que, se necesita conocer el resultado del primer problema, porque el segundo y el tercer problema no presentan explícitamente uno de sus datos numéricos. En su conjunto, se considera un problema aditivo de más de una etapa conformado por tres problemas en los que se identifican estructuras simples que constituyen a una estructura semántica compuesta de cambio 1-cambio 2-cambio 4 (C1-C2-C4).

Estos problemas se pueden representar por medio del siguiente esquema (ver Figura 3), que ayudan al proceso de lectura y comprensión del enunciado, así como la organización de los elementos e identificar la acción que ejerce una cantidad sobre otra.

Figura 3. Esquemas de los problemas propuestos en la consigna 1 del desafío 13.

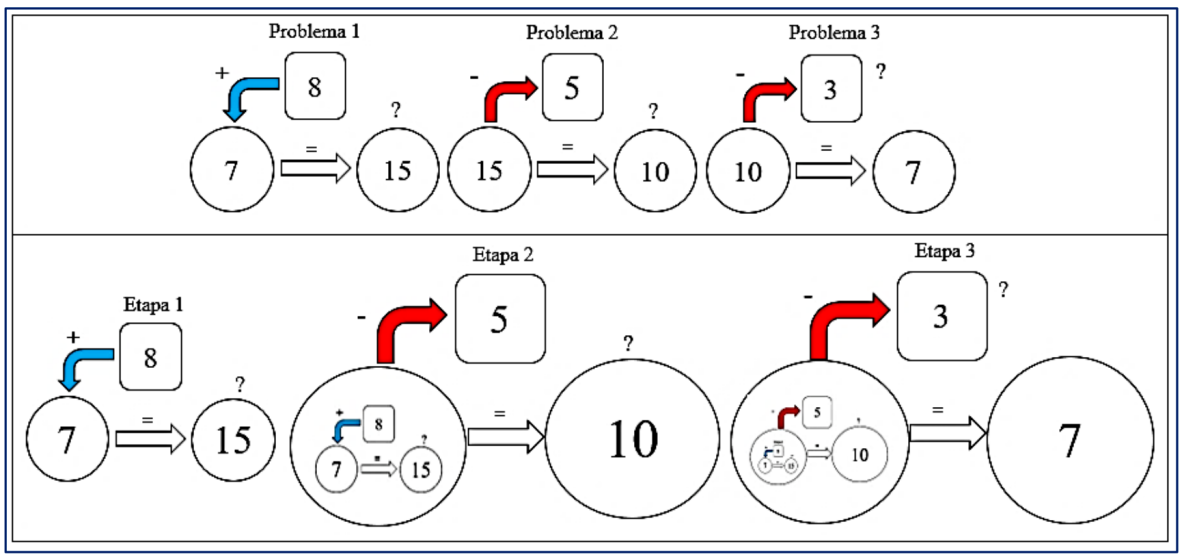

En la segunda consigna, se evidencian dos problemas aditivos. El primero se constituye de una estructura semántica de combinación 1 con la incógnita en la cantidad total. El segundo problema es compuesto o incluye más de una etapa, situación que requiere de tres etapas para su resolución. En la primera 
etapa se encuentra un problema con estructura semántica de cambio 1 aumento con incógnita en la cantidad final. Posteriormente, en la segunda etapa se parte del resultado obtenido anteriormente para poder resolver una situación problema con estructura semántica de cambio 2 disminución, con la incógnita en la cantidad final, y en la última etapa se presenta una estructura semántica de cambio 1 aumento con incógnita en la cantidad final.

Figura 4. Esquemas de los problemas propuestos en la consigna 2 del desafío 13.

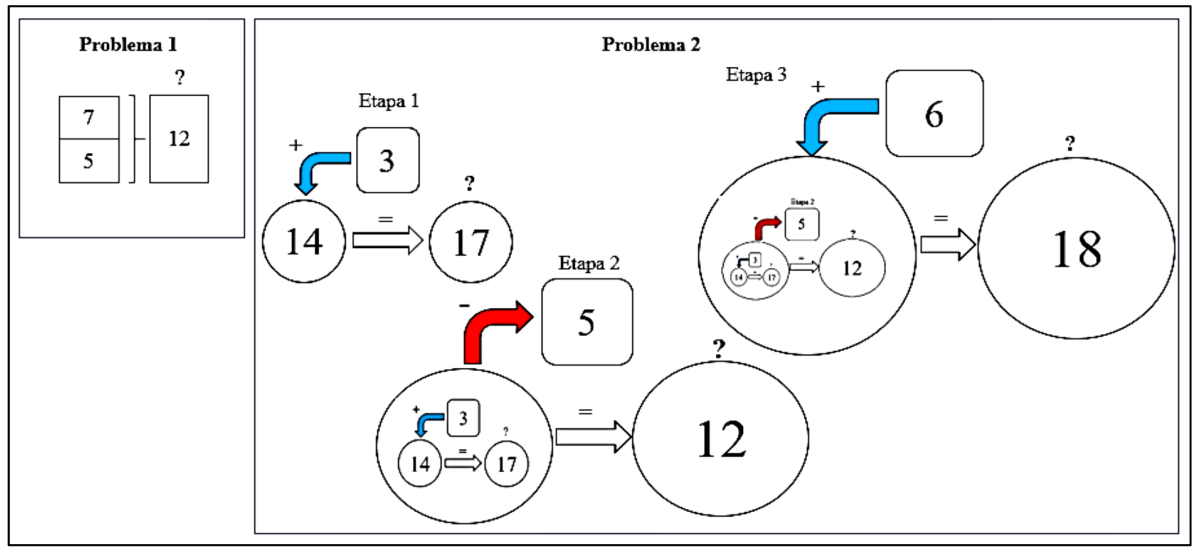

En la Figura 4 se evidencian los esquemas de los problemas 1 de una etapa y el problema 2 de tres etapas que conforma una estructura semántica compuesta cambio 1-cambio 1-cambio 2 (C1-C1-C2). De manera general, en la Tabla 4 se observa la cantidad de estructuras que se evidenciaron en todos los problemas aditivos del libro de primer grado.

Tabla 4. Estructuras semánticas simples en los PAEV aditivos del libro de primer grado.

\begin{tabular}{cccc}
\hline \multirow{4}{*}{ Estructura semántica } & PAEV aditivos en el libro de texto de primer grado & \\
& Componente sintáctica & Cantidad de estructuras & Porcentaje \% \\
\hline \multirow{3}{*}{ Cambio } & Cambio 1 & 17 & $40.5 \%$ \\
& Cambio 2 & 11 & $26.2 \%$ \\
Combinación & Cambio 4 & 1 & $2.4 \%$ \\
\hline Comparación & Combinación 1 & 10 & $23.8 \%$ \\
\hline Total & Comparación 1 & 3 & $7.1 \%$ \\
\hline
\end{tabular}




\subsection{ANÁLISIS DE LOS PAEV ADITIVOS DEL LIBRO DE SEGUNDO GRADO}

En particular, en este libro de texto se empiezan a proponer estructuras diferentes a las encontradas en el libro de primer grado, caracterizándose por mostrar variedad en el lugar de la incógnita, por ejemplo, en la cantidad inicial y la que modifica, para los problemas con estructura de cambio. Asimismo, la incógnita en una de las partes para los problemas de combinación y aparecen con más frecuencia los problemas de comparación y por primera vez los de igualación. En la Tabla 5 se presentan los desafíos considerados.

Tabla 5. Desafíos que contienen problemas aditivos en el libro de segundo grado.

\begin{tabular}{cccc}
\hline Bloque & Desafí & Consignas & Título \\
\hline II & 20 & 1 & El más rápido \\
\hline \multirow{2}{*}{$\|$} & 22 & 1 & ¿Qué debo hacer? \\
& 23 & 1 & ¿Cuál es la diferencia? \\
\multirow{2}{*}{ III } & 31 & 2 & La tienda de juguetes \\
& 33 & 2 & La ferretería \\
\hline \multirow{2}{*}{ IV } & 43 & 1 & ¿Cómo le hizo? \\
\cline { 2 - 4 } & 48 & 1 & ¿Cuál eliges? \\
\hline
\end{tabular}

Así, por ejemplo, el desafío veintitrés, está conformado por una consigna constituida por cinco problemas aditivos de enunciado verbal como se presentan en la Figura 5. Asimismo, corresponde al contenido: Resolución de problemas de sustracción en situaciones correspondientes a distintos significados: complemento, diferencia. 
Figura 5. Desafío 23, ¿Cuál es la diferencia? (SEP, 2016b, p.72).

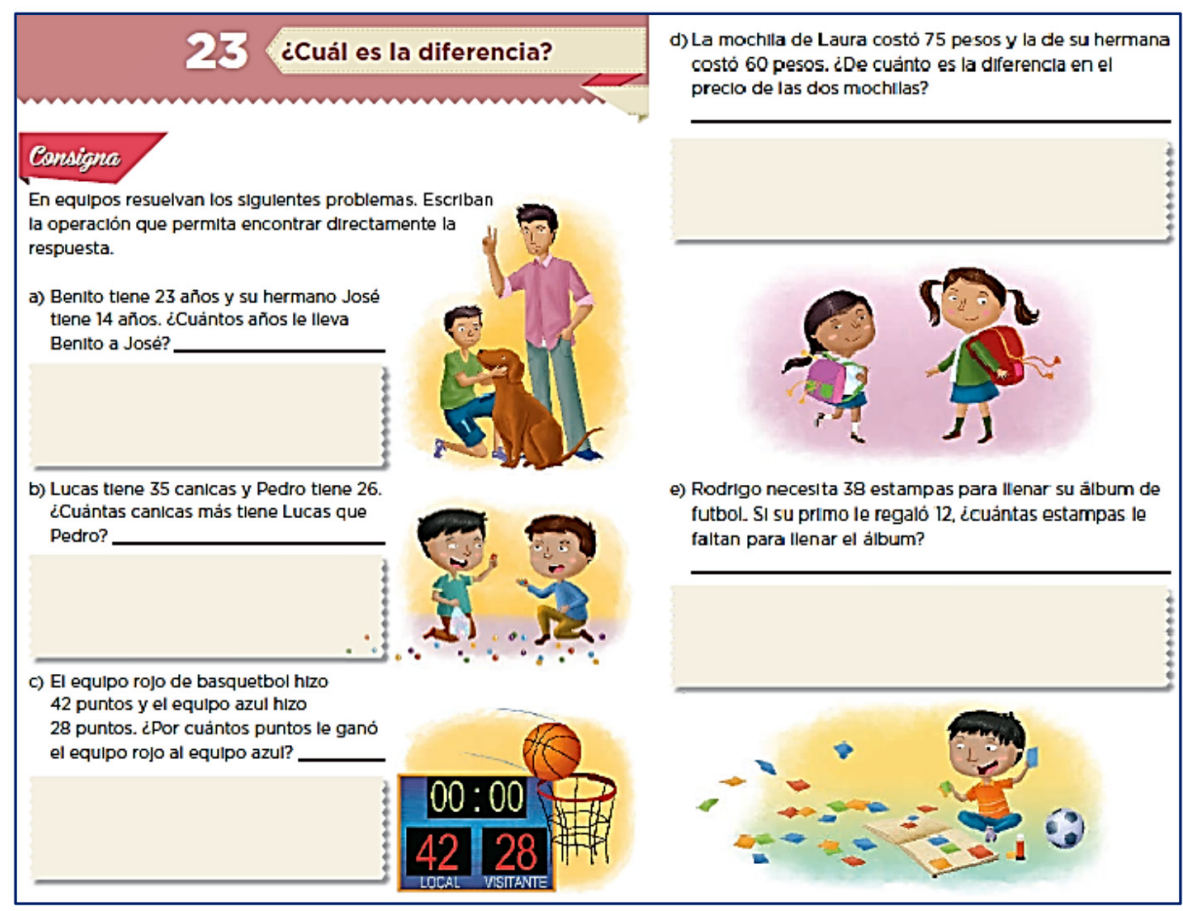

En el inciso a) se presenta un problema con estructura semántica de comparación 1 con la incógnita en la diferencia, es de una etapa y de tipo consistente. En el caso del inciso b) se propone un problema con estructura semántica de comparación 1 con la incógnita en la diferencia, es de una etapa o simple, con un enunciado consistente. En el inciso c) se presenta un problema con estructura semántica de comparación 1 con incógnita en la diferencia, de una etapa y de tipo consistente. Por su parte, el problema propuesto en el inciso d) por la redacción, podría tener implícitamente una estructura semántica de comparación que puede ser de comparación 1 o comparación 2. Sin embargo, por la forma como están ubicadas las cantidades numéricas en el enunciado, es un problema con estructura semántica de comparación 1 con incógnita en la diferencia.

El último inciso e) propone un problema constituido por una estructura semántica de combinación 2 con la incógnita en una de las partes que conforman el todo y es de una etapa de tipo consistente, ver Figura 6. 
Figura 6. Esquemas de los problemas propuestos en el desafío 23.

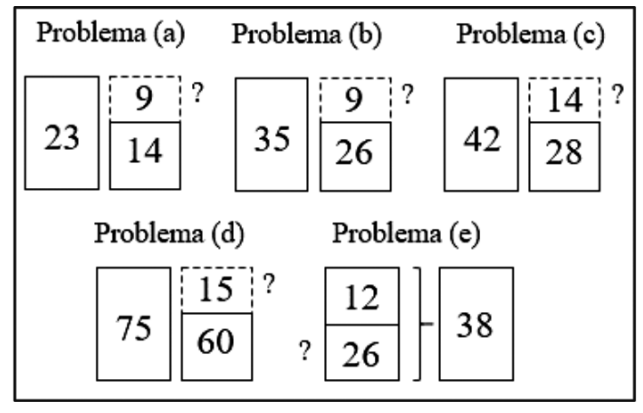

En este libro de texto, se logra evidenciar que la estructura semántica de cambio se completa teniendo en cuenta el problema con estructura de cambio 4 que aparece en el libro de primer grado. De igual manera sucede con la estructura de combinación, y para las estructuras de comparación e igualación sólo se presentan tres tipos atendiendo a su componente sintáctica. En este sentido, se puede afirmar que, los problemas conservan un tipo de estructuras sencillas pero que van aumentado su grado de dificultad a medida que van pasando de un grado escolar a otro. En la Tabla 6, se presentan todas las estructuras semánticas presentes en los problemas del libro de segundo grado.

Tabla 6. Estructuras semánticas simples en los PAEV aditivos del libro de segundo grado.

\begin{tabular}{cccc}
\hline \multirow{4}{*}{ Estructura semántica } & PAEV aditivos en el libro de texto de segundo grado & \\
\hline \multirow{3}{*}{ Cambio } & Componente sintáctica & Cantidad de estructuras & Porcentaje \% \\
& Cambio 1 & 2 & $5 \%$ \\
& Cambio 2 & 3 & $7.5 \%$ \\
& Cambio 3 & 2 & $5 \%$ \\
& Cambio 5 & 4 & $10 \%$ \\
Combinación & Cambio 6 & 2 & $5 \%$ \\
& Combinación 1 & 17 & $42.5 \%$ \\
& Combinación 2 & 4 & $10 \%$ \\
\hline \multirow{2}{*}{ Comparación } & Comparación 1 & 4 & $10 \%$ \\
\hline Igualación & Comparación 3 & 1 & $2.5 \%$ \\
Total & Igualación 1 & 1 & $2.5 \%$ \\
\hline
\end{tabular}




\subsection{ANÁLISIS DE LOS PAEV ADITIVOS DEL LIBRO DE TERCER GRADO}

En el libro de tercer grado se identificaron desafíos que contienen PAEV aditivos con estructuras semánticas sencillas, como las de cambio 1 y combinación 2, estructuras complejas como comparación 1 e igualación 1 (ver Figura 7). Cabe resaltar que, los problemas de este libro contienen combinaciones de estructuras, tanto aditivas como multiplicativas, por lo que en algunos problemas analizados sólo se tomó en cuenta la parte que implica una adición y/o sustracción.

Tabla 7. Desafíos que contienen problemas aditivos en el libro de tercer grado.

\begin{tabular}{cccc}
\hline Bloque & Desafío & Consignas & Título \\
\hline I & 4 & 2 & Rapidez mental \\
& 13 & 3 & Elaboración de galletas \\
III & 43 & 3 & Sumas y restas \\
\hline IV & 55 & 1 & La fiesta \\
\cline { 2 - 4 } & 56 & 1 & ¿Cuál de todas? \\
\hline
\end{tabular}

En este sentido, el desafío 43 está conformado por tres consignas, de las cuales se tomó la primera a manera de ejemplo, en la que se proponen cuatro PAEV aditivos (ver Figura 7), pertenecientes al contenido: determinación y afirmación de un algoritmo para la sustracción de números de dos cifras. 
Figura 7. Desafío 43, sumas y restas (SEP, 2016c, p.142).

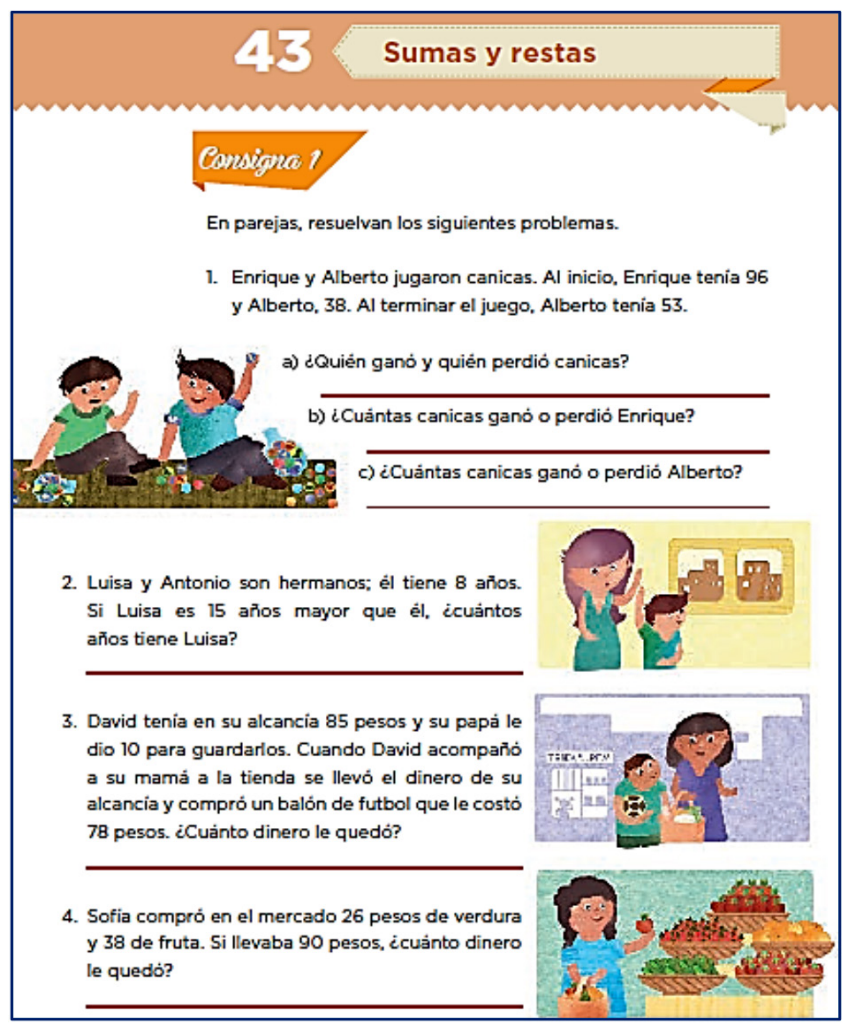

En cuanto a la primera consigna (ver Figura 7), el primer problema está constituido por dos estructuras semánticas, una de cambio 3 y la otra de cambio 4, ambas con la incógnita en la cantidad de cambio, identificadas para dar respuesta a los incisos a, b y c. Es un problema que requiere de dos etapas con estructura semántica compuestas cambio 3-cambio 4 (C3-C4). El segundo problema tiene una estructura semántica de comparación 3 con la incógnita en el comparado. Además, es de una etapa, de tipo consistente El tercer problema es de dos etapas, en la primera se presenta una estructura semántica de cambio 1 con la incógnita en la cantidad final. En la segunda etapa se evidencia una estructura semántica de cambio 2 con la incógnita en la cantidad final, es de tipo consistente y tiene una estructura semántica compuesta cambio 1-cambio 2 (C1-C2). Ver esquemas en la Figura 8. 
Figura 8. Esquemas de los problemas 1, 2 y 3 del desafío 43.

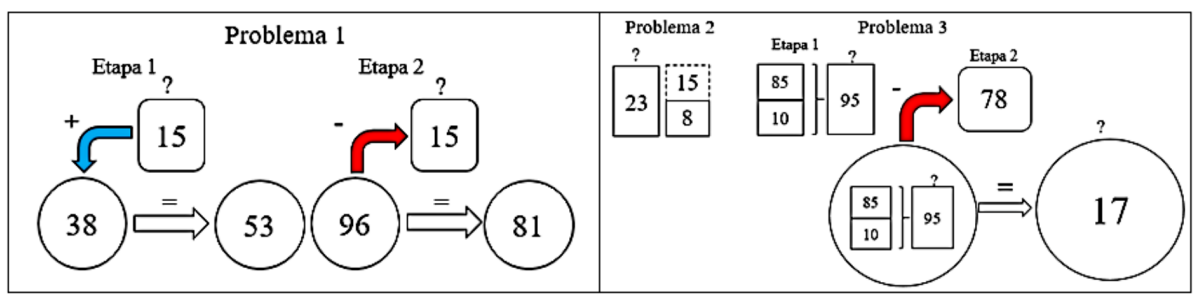

El cuarto problema es de dos etapas, en la primera etapa se presenta una estructura semántica de combinación 1 con la incógnita en la cantidad total, y en la segunda etapa se identificó una estructura semántica de cambio 2 con la incógnita en la cantidad final. Este problema es de tipo consistente, y tiene una estructura semántica compuesta de combinación 1- cambio 2 (CB1-C2), presentándose a través de esquemas en la Figura 9.

Figura 9. Esquemas del problema 4 del desafío 43.

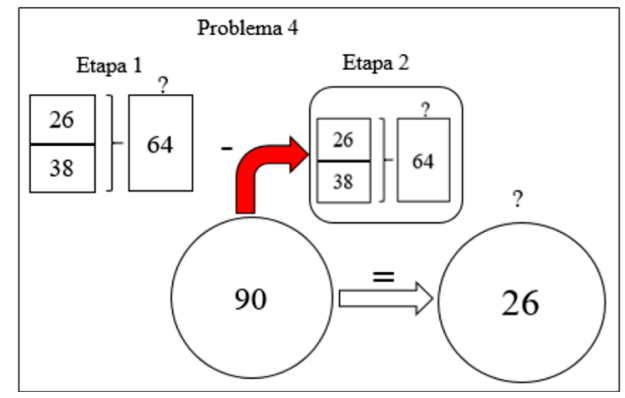

En relación con la consigna 3, se evidencia un problema de tres etapas. La primera se conforma de tres subetapas. La primera y segunda subetapa tienen una estructura semántica de combinación 1 con la incógnita en la cantidad total, en la tercera subetapa se presenta una estructura semántica de cambio 2 con la incógnita en la cantidad final. En la segunda etapa se presenta una estructura semántica de combinación 1 con la incógnita en la cantidad total. En su conjunto estos problemas son de tipo consistentes. En la tercera etapa se identificó una estructura semántica de combinación 2 con la incógnita en una de las partes que conforman el todo, ver Figura 10. 
Figura 10. Esquema del problema contenido en la consigna 3 del desafío 43.

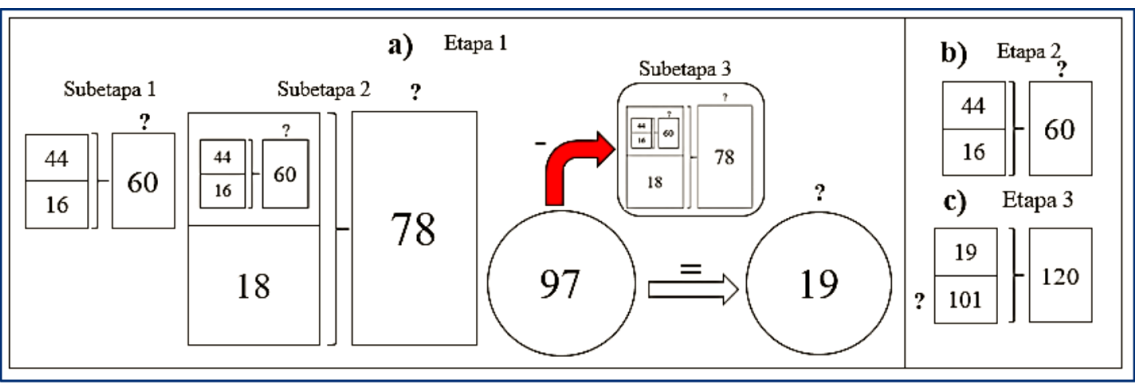

A su vez, se identificaron problemas conformados por estructuras semánticas compuestas, considerando la estructura de combinación 1 siendo la más frecuente en cada composición. También, se resalta un factor importante en la resolución de problemas inconsistentes presentándose casos en los que se involucran hasta tres etapas en las que la estructura semántica es la misma, en este caso cambio 5 e igualación 1.

Tabla 8. Estructuras semánticas simples en los PAEV aditivos del libro de tercer grado.

\begin{tabular}{cccc}
\hline \multirow{2}{*}{ Estructura semántica } & PAEV aditivos en el libro de texto de tercer grado & \\
\hline \multirow{2}{*}{ Cambio } & Componente sintáctica & Cantidad de estructuras & Porcentaje \% \\
& Cambio 1 & 4 & $11.4 \%$ \\
& Cambio 2 & 8 & $22.8 \%$ \\
& Cambio 3 & 1 & $2.9 \%$ \\
Combinación & Cambio 4 & 1 & $2.9 \%$ \\
Comparación & Combinación 1 & 3 & $8.6 \%$ \\
\hline Igualación & Combinación 2 & 11 & $31.4 \%$ \\
\hline Total & Comparación 3 & 3 & $8.6 \%$ \\
\hline
\end{tabular}


En la Tabla 8 se sintetizan las estructuras semánticas contenidas en los PAEV aditivos en el libro de tercer grado. Con base en esta información, se da a conocer que las estructuras semánticas más identificadas son las de combinación 1 con la incógnita en la cantidad total, 31.4\% de los problemas. Seguidos por las estructuras de cambio 2 encontradas, 22.8\%. Asimismo, se presenta con 11.4\% las estructuras de cambio 1 y 8.6\% las estructuras de cambio 5, combinación 2 e igualación 1, evidenciándose para cada tipo de problema tres estructuras semánticas. En menor frecuencia se presentan las estructuras de cambio 3, cambio 4 y comparación 3,2.9\%.

\subsection{ESTRUCTURAS SEMÁNTICAS COMPUESTAS EN LOS PAEV ADITIVOS}

Con base en las estructuras semánticas simples que se identificaron en los problemas, a continuación, se presentan las estructuras semánticas compuestas evidenciadas en los PAEV aditivos en los tres libros de textos analizados, en los que se requieren para su resolución más de una operación (adición y/o sustracción), ver Tabla 9.

Tabla 9. PAEV aditivos con estructuras semánticas compuestas en libros de texto de primero, segundo y tercer grado.

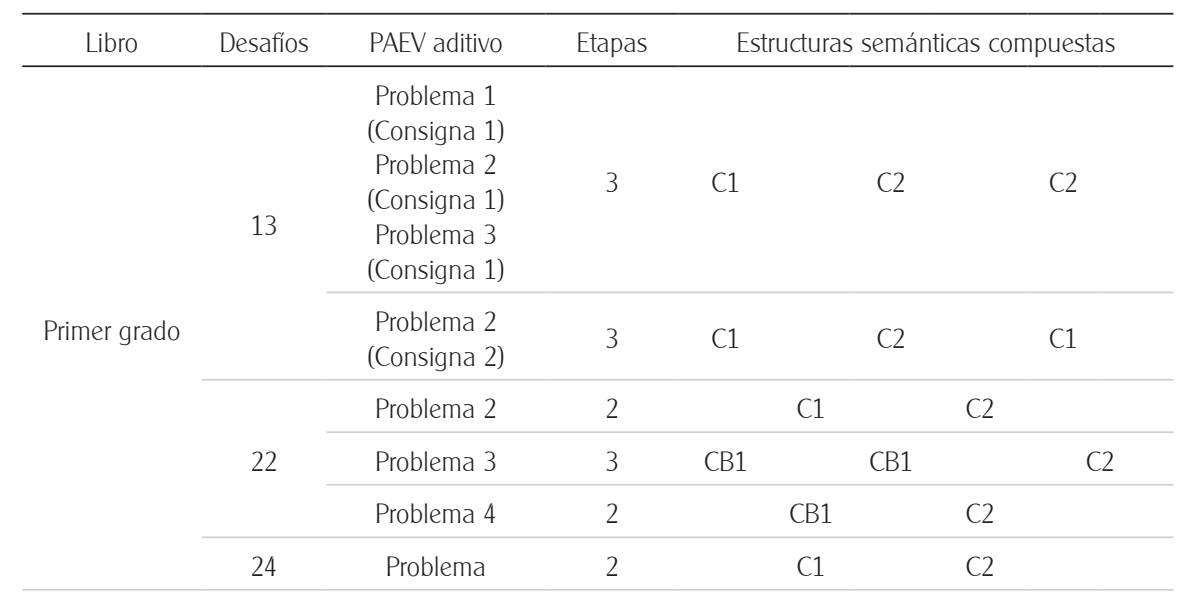




\begin{tabular}{|c|c|c|c|c|c|c|c|c|}
\hline \multirow{7}{*}{ Primer grado } & \multirow{3}{*}{36} & Problema 1 & 2 & \multicolumn{3}{|c|}{ CB1 } & CB1 & \multirow[b]{2}{*}{ C2 } \\
\hline & & Problema 2 & 3 & CB1 & & CB1 & & \\
\hline & & Problema 3 & 4 & & B1 & CB1 & CB1 & $\mathrm{C} 1$ \\
\hline & \multirow{4}{*}{46} & Problema 1 & 2 & \multicolumn{3}{|c|}{$\mathrm{C} 2$} & $\mathrm{C} 2$ & \\
\hline & & Problema 2 & 3 & $\mathrm{C} 1$ & & $\mathrm{C} 1$ & & $\mathrm{C} 1$ \\
\hline & & Problema 3 & 3 & $\mathrm{CP} 1$ & & CP1 & & $\mathrm{CP} 1$ \\
\hline & & Problema 4 & 2 & C & & & $\mathrm{C} 1$ & \\
\hline \multirow{5}{*}{$\begin{array}{l}\text { Segundo } \\
\text { grado }\end{array}$} & \multirow{2}{*}{31} & $\begin{array}{l}\text { Problema (a) } \\
\text { (Consigna 1) }\end{array}$ & 2 & \multicolumn{2}{|c|}{ CB1 } & & $\mathrm{C} 6$ & \\
\hline & & $\begin{array}{l}\text { Problema (c) } \\
\text { (Consigna 2) }\end{array}$ & 2 & \multicolumn{2}{|c|}{ CB1 } & & $\mathrm{C} 5$ & \\
\hline & 43 & Problema (b) & 6 & $\mathrm{C} 2$ & CB1 & CB1 & CB1 & CB1 CB1 \\
\hline & \multirow{2}{*}{48} & Problema (a) & 3 & \multicolumn{2}{|c|}{ CB1 } & CB1 & & $\mathrm{C} 2$ \\
\hline & & Problema (g) & 2 & \multicolumn{3}{|c|}{ CB1 } & CB2 & \\
\hline \multirow{12}{*}{ Tercer grado } & \multirow{2}{*}{13} & Problema 1 & 2 & \multicolumn{3}{|c|}{$\mathrm{Cl}$} & $\mathrm{C} 1$ & \\
\hline & & Problema 2 & 3 & \multicolumn{2}{|c|}{$\mathrm{C} 5$} & C5 & & C5 \\
\hline & \multirow{5}{*}{43} & $\begin{array}{c}\text { Problema } 1 \\
\text { (Consigna 1) }\end{array}$ & 2 & \multicolumn{3}{|c|}{$\mathrm{C} 3$} & $\mathrm{C} 4$ & \\
\hline & & $\begin{array}{l}\text { Problema } 3 \\
\text { (Consigna 1) }\end{array}$ & 2 & \multicolumn{3}{|c|}{$\mathrm{C} 1$} & $\mathrm{C} 2$ & \\
\hline & & $\begin{array}{l}\text { Problema } 4 \\
\text { (Consigna 1) }\end{array}$ & 2 & \multicolumn{3}{|c|}{ CB1 } & $\mathrm{C} 2$ & \\
\hline & & \multirow{2}{*}{$\begin{array}{c}\text { Problema } 1 \\
\text { (Consigna 3) }\end{array}$} & \multirow{2}{*}{3} & CB1 & CB1 & & & \\
\hline & & & & \multicolumn{2}{|c|}{$\mathrm{C} 2$} & CB1 & & CB2 \\
\hline & 55 & Problema 1 & 3 & \multicolumn{2}{|c|}{ CB1 } & CB1 & & CB2 \\
\hline & \multirow{4}{*}{56} & Problema 1 & 2 & \multicolumn{3}{|c|}{ CB1 } & CB1 & \\
\hline & & Problema 2 & 2 & \multicolumn{2}{|c|}{ CB1 } & CB1 & & CB2 \\
\hline & & Problema 3 & 2 & \multicolumn{3}{|c|}{ CB1 } & & \\
\hline & & Problema 4 & 3 & \multicolumn{2}{|c|}{ IG1 } & IG1 & & IGI \\
\hline
\end{tabular}




\section{DISCUSIÓN Y CONCLUSIONES}

En esta investigación se encontró que la mayoría de los PAEV aditivos propuestos en los libros de texto correspondientes al segundo periodo escolar de la educación básica en México, presentan estructuras semánticas consideradas sencillas de resolver, tales como cambio 1, 2 y combinación 1 y pocos problemas con estructuras de comparación e igualación, como lo reportan Orrantia et al. (2005), Aguillón y Resendiz (2012) y Chamoso et al. (2014). Por tanto, es importante sugerir al profesor realizar un análisis previo de las tareas a abordar, con el propósito de conocer y evaluar la pertinencia de los mismos (Font y Godino, 2006).

El análisis realizado permitió identificar PAEV aditivos estructurados por más de una etapa, que contienen varias estructuras semánticas. Lo anterior no quiere decir que los problemas sean desafiantes por presentar enunciados extensos o más datos. Es importante resaltar esta situación, puesto que los problemas con las características mencionadas, en su mayoría contienen estructuras de cambio 1, cambio 2, combinación 1, las que no permiten evidenciar un grado elevado de dificultad o desafío, en consecuencia, la promoción de problemas genuinos es escasa. A su vez, las características semánticas y sintácticas de los problemas influyen en las formas de resolución del estudiante, es decir, si no se enfatiza en comprender e identificar la estructura semántica del problema, se podría elegir un procedimiento inadecuado (Butto y Martínez, 2012).

Con base en los resultados obtenidos en este trabajo, se constata que los niveles bajos en la competencia de resolución de problemas por parte de los estudiantes podrían estar influenciados por la forma como se promueven los problemas en los libros de texto, los cuales son usados de manera directa por el profesor durante su planificación (Jaime, Chapa y Gutiérrez, 1992 y Aké y Godino, 2018).

El uso del modelo teórico adoptado en este trabajo fue relevante, debido a que permitió categorizar los problemas de acuerdo con su estructura semántica y componente sintáctica (Riley et al., 1983; Puig y Cerdán, 1988; Orrantia et al., 2005; Cañadas y Castro, 2011). Asimismo, permitió esquematizar los problemas aludiendo a representaciones gráficas para organizar y operar con los datos involucrados. Esta forma de proceder en la resolución de problemas aditivos, es fundamental para la etapa inicial de la educación básica, pues coadyuva a la comprensión del mismo problema, así como a organizar datos para identificar la operación que permitirá establecer la relación aditiva adecuada. En este sentido, los esquemas identificados en esta investigación, se conectan directamente con las tipologías de problemas de acuerdo con Nesher (1999), ubicando a los problemas sencillos en procesos de 
conteo y cambio, mientras que los problemas desafiantes ameritan esquemas parte-todo donde se evidencian procesos de comparación y reversibilidad.

Por otra parte, en el plan y programas de estudios se afirma que al culminar el segundo periodo escolar los estudiantes saben resolver problemas aditivos con diferentes estructuras (SEP, 2011a), pero los resultados de este trabajo evidencian ausencia respecto de la presencia del total de estructuras semánticas, en particular las que involucran problemas desafiantes. Cabe resaltar que, aunque los libros de texto no fueron diseñados a partir de los elementos teóricos usados en este trabajo, dichos elementos permiten identificar problemas aditivos sencillos y desafiantes en los documentos curriculares oficiales (plan de estudios, programas de estudio y libros de texto), dando oportunidad a los docentes de atender la resolución de PAEV aditivos con las diferentes estructuras.

El análisis de contenido desarrollado es útil para profesores de matemáticas de primaria como herramienta para clasificar los diferentes PAEV aditivos, considerando como modelo de enseñanza para promover los mismos desde los más sencillos a los más dificultosos o viceversa, pero haciendo énfasis en la estructura semántica y la componente sintáctica. En este sentido, la forma de esquematizar es otro elemento que contribuye en la comprensión y representación del problema.

\section{REFERENCIAS}

Aguillón, M. y Resendiz, A. (2012). Identificación y clasificación de problemas aditivos en los libros de texto del segundo ciclo de Educación primaria (Tesis de pregrado). Universidad Pedagógica Nacional, México, D.F.

Aké, L. P. y Godino, J. D. (2018). Análisis de tareas de un libro de texto de primaria desde la perspectiva de los niveles de algebrización. Educación matemática, 30(2), 171-201. https://dx.doi.org/10.24844/em3002.07

Azcárate, P. y Serradó, A. (2006). Tendencias didácticas en los libros de texto de matemáticas para la ESO. Revista de Educación, 340, 341- 378.

Bermejo, V. y Rodríguez, P. (1987). Estructura semántica y estrategias infantiles en la solución de problemas verbales de adición. Infancia y aprendizaje, 39(40), 71-81.

Bonilla, M., Sánchez, N. y Guerrero, F. (1999). Estructura aditiva y formación de profesores para la educación básica. En Bonilla, M., Sánchez, N., Vidal, M., Guerrero, F., Lurduy, J., Romero, J., Rojas, P., Mora, L., y Barón, C. (Eds), La enseñanza de la aritmética escolar y la formación del profesor. (pp.1-150). Bogotá, Colombia: Grupo Editorial Gaia.

Butto, C. y Martínez, C. (2012). Abordaje basado en competencias: la resolución de problemas aditivos en el nivel básico. Revista horizontes pedagógicos, 14(1), 30-42. 
Blanco, L., Caballero, A. y Cárdenas, J. (2015). Los problemas aritméticos escolares. En L. J. Blanco, J. A. Cárdenas y A. Caballero (Eds.), La resolución de problemas de Matemáticas en la formación inicial de profesores de primaria (pp. 123-138). España: Universidad de Extremadura.

Cañadas, M. C. y Castro, E. (2011). Aritmética de los números naturales. Estructura aditiva. En Segovia y Rico (Coord.). Matemáticas para maestros en Educación Primaria (pp. 75-98). Madrid: Pirámide.

Carpenter, T., Hiebert, J. y Moser, J. (1981). Problem structure and first-grade children's initial solution processes for simple addition and subtraction problems. Journal for research in mathematics education, 2(1), 27-39.

Castro, A. (2013). Contribución al análisis de la estructura semántica de los problemas aritméticos elementales. (Tesis de maestría). Universitat Autònoma de Barcelona, Barcelona.

Castro, A., Gorgorió, N. y Prat, M. (2014). Indicios verbales en los PAEV aditivos planteados por estudiantes para maestro. En M. T. González, M. Codes, D. Arnau y T. Ortega (Eds.), Investigación en Educación Matemática XVIII (pp. 217-226). Salamanca: SEIEM.

Chamoso, J. M., Vicente, S., Manchado, E. y Múñez, D. (2014). Los Problemas de Matemáticas Escolares de Primaria, ¿Son solo Problemas para el aula? Cuadernos de Investigación y Formación en Matemática, 9, 261-279.

Common Core State Standars Initiative. (2018). Common core state standars for mathematics. Washington, D.C: National Governors Association Center for Best Practices and the Council of Chief State School Officers.

Durand, C. y Vergnaud, G. (1976). Structures Addictives et complexité psychogénétique. Revue française de pédagogie, 36, 28-43.

Echenique, I. (2006). Matemáticas resolución de problemas. Navarra: Fondo de publicaciones del gobierno de Navarra.

Font, V. y Godino, J. D. (2006). La noción de configuración epistémica como herramienta de análisis de textos matemáticos: su uso en la formación de profesores. Educaçao Matemática Pesquisa, 8 (1), 67-98.

García, S.R. (2011). Resolución de problemas matemáticos en la escuela primaria: Proceso representacional, didáctico y evaluativo. México, D.F.: Trillas.

García-García, J. (2014). Los problemas aritméticos en primaria: un estudio sobre las concepciones de profesores bilingües. NÚMEROS, 87, 37-58.

García-García, J., Navarro, C., y Rodríguez-Vásquez, F. (2014). La resolución de problemas en un contexto Nuuu Savi: un estudio de casos con niños de sexto grado de primaria. Educación matemática, 26(1), 127-152.

Jaime, A., Chapa, F. y Gutiérrez, A. (1992). Definiciones de triángulos y cuadriláteros: errores e inconsistencias en libros de texto de E.G.B. Epsilon, 23, 49-62. 
Hegarty, M., Mayer, R. E. y Monk, C. A. (1995). Comprehension of arithmetic word problems: A comparison of successful and unsuccessful problem solvers. Journal of Educational Psychology, 87, 18-32.

Heller, J. I. y Greeno, J. G. (1978). Semantic processing in arithmetic word problem solving. Paper presented at the Midwestern Psychological Association Convention, Chicago.

Instituto Nacional para la Evaluación de la Educación (2016). Manual para la aplicación, clasificación, análisis y uso de los resultados de la prueba Planea diagnóstica 20162017. Recuperado de http://planea.sep.gob.mx/content/ba_d/docs/2017/MANUAL_ PLANEA_DIAGNOSTICA_2017.pdf

López-Noguero, F. (2002). El análisis de contenido como método de investigación. Revista de Educación, 4,167-179.

National Council of Teachers of Mathematics. (2000). Principles and standards for school mathematics. Reston, VA: National Council of Teachers of Mathematics.

Navarro, C. (2015). Libros de texto gratuito de matemáticas, reforma 2011: el caso de los números naturales y números fraccionarios (Tesis doctoral). Universidad Autónoma de Guerrero, Chilpancingo, Gro.

Nesher, P. (1999). El papel de los esquemas en la resolución de problemas de enunciado verbal. SUMA, 31, 19-26.

Nesher, P., Greeno, J. y Riley, M. (1982). The development of semantic categories for addition and subtraction. Educational studies in mathematics, 13, 373-394.

OCDE. (2016). Nota país. Programa para la evaluación internacional de alumnos (PISA). PISA 2015-resultdos. México.

Orrantia, J. (2003). El rol del conocimiento conceptual en la resolución de problemas aritméticos con estructura aditiva. Infancia y aprendizaje, 26(4), 451-468.

Orrantia, J., González, L. y Vicente, S. (2005). Un análisis de los problemas aritméticos en Ios libros de texto en educación primaria. Infancia y aprendizaje, 28(4), 429-451.

Orrantia, J. (2006). Dificultades en el aprendizaje de las matemáticas: una perspectiva evolutiva. Revista psicopedagogía, 23(71), 158-180.

Ortíz, C. (2014). Procedimiento de resolución de problemas aditivos escolares en el contexto de compra-venta en niños de segundo grado (Tesis de maestría). Universidad Autónoma de Querétaro, Querétaro.

Pepin, B., Gueudet, G., y Trouche, L. (2013). Investigating textbooks as crucial interfaces between culture, policy and teacher curricular practice. Two contrasted case studies in France and Norway. ZDM The International Journal of Mathematics Education, 45, 685-698.

Polotskaia, E., Savard, A. y Freiman, V. (2016). Investigating a case of hidden misinterpretations of an additive word problem: structural substitution. European journal of psychology of education, 31(2), 135-153. 
Pólya, G. (1989). Cómo plantear y resolver problemas. México: Editorial Trillas.

Puig, L. y Cerdán, F. (1988). Problemas aritméticos escolares. Síntesis.

Real Academia Española. (2014). Diccionario de la lengua española (23. Ed.). Madrid, España: Autor.

Riley, M. y Greeno, J. (1988). Developmental Analysis of Understanding Language About Quantities and of Solving Problems. Cognition and Instruction, 5(1), 49-101.

Riley, M., Greeno, J. y Heller, J. (1983). Development of children's problem-solving ability in arithmetic. En H. Ginsburg (Ed.), The development of mathematical thinking (pp. 153-196). Nueva York: Academic Press.

Rizo, C. y Campistrous, L. (1999). Estrategias de resolución de problemas en la escuela. Revista Latinoamericana de Investigación en Matemática Educativa, 2(3), 31-45.

Sánchez, M. y Vicente, S. (2015). Models and processes for solving arithmetic word problems proposed by Spanish mathematics textbooks. Culture and education, 27(4), 695-725.

Secretaría de Educación Pública (2011a). Programas de estudios 2011 Guía para el maestro. Educación Básica. México: SEP.

Secretaría de Educación Pública (2011b). Plan de estudios 2011. Educación Básica. México: SEP.

Disponible en: http://issuu.com/dgeb/docs/planedu2011?e=3503076/2622744

Secretaría de Educación Pública (2016a). Desafíos matemáticos. Libro para el maestro, Primer grado. México: SEP.

Secretaría de Educación Pública (2016b). Desafíos matemáticos. Libro para el maestro, Segundo grado. México: SEP.

Secretaría de Educación Pública (2016c). Desafíos matemáticos. Libro para el maestro, Tercer grado. México: SEP.

Schoenfeld, A. (1985). Mathematical problem solving. Orlando, Florida: Academic Press. Van Dijk, T. y Kintsch, W. (1983). Strategies of discourse comprehension. New York: Academic Press.

Vergnaud, G. (1991). El niño, las Matemáticas y la realidad. México: Trillas.

Verschaffel, L. y De Corte, E. (1993). A decade of Research on world problem solving in leuven: theoretical, methodological, and practical outcomes. Educational psychology review, 5(3), 239-256.

Verschaffel, L., Greer, B. y De Corte, E. (2000). Making sense of word problems. Lisse, The Netherlands: Swets \& Zeitlinger Publishers.

CAMILO RODRÍGUEZ

Dirección: Colonia La Haciendita, Avenida Médico Militar, Código Postal, 39087, Chilpancingo de los Bravo, México.

Teléfono: 7471842676;

Correo electrónico: camilorodriguez274@gmail.com 